\title{
Capacidade de suporte de cargas de um Latossolo submetido a duas alternativas de uso do solo e sob Floresta Nativa
}

\author{
Load support capacity of a Oxisol submitted to two alternatives of land \\ use and under natural forest
}

\author{
André Ribeiro Silveira ${ }^{1}$ (1), Wellington Willian Rocha ${ }^{2}$ (iD, Ângelo Márcio Pinto Leite ${ }^{2}$ (1), \\ Josiel de Jesus Santos ${ }^{1}$ (D), Moacir de Souza Dias Júnior ${ }^{1}$ (D) \\ ${ }^{1}$ Universidade Federal de Lavras - UFLA, Lavras, MG, Brasil \\ ${ }^{2}$ Universidade Federal dos Vales do Jequitinhonha e Mucuri - UFVJM, Diamantina, MG, Brasil
}

\begin{abstract}
Como citar: Silveira, A. R., Rocha, W. W., Leite, Â. M. P., Santos, J. J., \& Dias Júnior, M. S. (2022). Capacidade de suporte de cargas de um Latossolo submetido a duas alternativas de uso do solo e sob Floresta Nativa. Scientia Forestalis, 50, e3731. https://doi.org/10.18671/scifor.v50.05
\end{abstract}

\begin{abstract}
Resumo
Um dos principais fatores da degradação do solo é a compactação, cujas causas incluem práticas florestais e agrícolas inadequadas. O tipo e a intensidade desse manejo alteram a estrutura do solo e comprometem a qualidade física dessas áreas. A pressão de pré-consolidação (PP) constitui uma ferramenta eficaz e importante alternativa na análise da compactação do solo. Nesse sentido, objetivouse, com esse trabalho, analisar a capacidade de suporte de cargas de um latossolo submetido a duas alternativas de uso do solo e sob Floresta Nativa, em Curvelo, MG. Foram coletadas amostras indeformadas e deformadas do Cerrado (CE), do sistema Integração Lavoura Pecuária Floresta (ILPF) e da Floresta Nativa (FN) para análises texturais e de matéria orgânica desses locais. Para a obtenção da pressão de pré-consolidação (PP) e a definição dos modelos de capacidade de suporte de carga do latossolo, as amostras foram submetidas ao ensaio de compressão uniaxial, utilizando-se um consolidômetro. As áreas de uso correspondente ao sistema ILPF e ao CE não apresentaram diferença significativa entre si quanto a PP, devido a ambas as áreas terem sofrido pisoteio animal e, ou tráfego de máquinas. Por outro lado, houve diferença significativa entre o sistema ILPF e o CE em relação a FN, tendo estes dois usos do solo apresentando uma maior PP. A explicação para o solo da FN ter apresentado uma menor PP deve-se à maior disponibilidade de matéria orgânica (MO) neste ambiente, por a MO promover uma diminuição na pressão de carga aplicada na estrutura do solo.
\end{abstract}

Palavras-chave: Compactação; Pré-consolidação; Solo.

\begin{abstract}
One of the main factors that contribute to the degradation of the soils around the world is compaction, whose causes include inadequate forest and agricultural practices. The type and intensity of these managements alter the structure of the soils and compromise the physical quality of the areas. The pre consolidation pressure (PP) is an efficient and important tool for the analysis of soil compaction. This study aims to analyze the Load Support Capacity of a Oxisol submitted to two alternative soil managements and to a natural forest, located in Curvelo, MG. Undeformed and deformed samples were collected in the areas of Cerrado (CE), Integration of Crop Livestock and Forestry area (ILPF) and Native Forest (FN) for analysis of texture and organic matter. To obtain the pre consolidation pressure (PP) and the definition of the Load Support Capacity models of the Oxisol, the samples were submitted to a uniaxial compression essay, utilizing a consolidometer. The areas corresponding to the ILPF and CE did not present significant differences between them in PP, because both of the areas have suffered animal
\end{abstract}

Fonte de financiamento: Nenhuma.

Conflito de interesse: Nada a declarar.

Autor correspondente: andre.silveira2@estudante.ufla.br

Recebido: 15 junho 2021.

Aceito: 11 janeiro 2022

Editor: Mauro Valdir Schumacher.

(c) (i) Este é um artigo publicado em acesso aberto (Open Access) sob a licença Creative Commons Attribution, que permite uso, distribuição e c. reprodução em qualquer meio, sem restrições desde que o trabalho original seja corretamente citado. 
stomping and or machinery traffic. On the other side, there was a significant difference between the ILPF system and the CE as for the FN soil, in which the first two land uses presented a higher PP. To explain why the FN presented a lower PP, the higher availability of organic matter (MO) in this environment must be taken into account, since the MO promotes less load pressure applied to the structure of the soil.

Keywords: Compaction; Load board pressure; Soil.

\section{INTRODUÇÃO}

O excesso de tráfego em áreas de cultivo e a utilização de máquinas inapropriadas podem provocar modificações importantes no solo, ocasionando consequências adversas como aumento da resistência à penetração das raízes, alteração na disponibilidade de fluxo e na capacidade de retenção da água e nutrientes, bem como redução da aeração do solo (Lima \& Leite, 2014; Epron et al., 2016; Cambi et al., 2017).

Assim, o uso intensivo do solo de maneira inadequada pode gerar grandes prejuízos, como a compactação (Secco et al., 2009; Kunz et al., 2013), que se resume à deformação volumétrica do solo e alteração nas suas características físicas, provocando uma mudança na relação água-solo (Rocha et al., 2007). Essa compactação pode estar relacionada com a porosidade e a densidade do solo, onde um solo com menor número de espaços vazios (poros) se torna mais denso (Tang et al., 2011), aumentando assim sua resistência à penetração e reduzindo o crescimento radicular das plantas, resultando na redução da produtividade das culturas (Silva et al., 2002; Gubiani et al., 2013).

O termo compactação do solo refere-se à compressão do solo não saturado, durante a qual ocorre um aumento da densidade, em consequência da redução de volume pela expulsão do ar (Dias Júnior \& Pierce, 1996). A compactação excessiva pode limitar a adsorção e/ou absorção de nutrientes, infiltração e redistribuição de água, trocas gasosas e desenvolvimento do sistema radicular (Smucker \& Erickson, 1989).

A intensidade de compactação do solo depende de vários fatores, que incluem a pressão exercida pelo maquinário utilizado, em virtude do tamanho e tipo dos rodados, peso, frequência do tráfego na área de cultivo (Lima \& Leite, 2014), textura, agregação e umidade do solo, sendo este último um dos fatores mais importantes no manejo do solo (Lopes et al., 2006, 2011).

Um superpastejo causa compactação, devido ao impacto do pisoteio intensivo sob altas taxas de lotação animal, podendo provocar uma perda considerável da cobertura do solo (Collares et al., 2011). Assim, um maior grau de compactação dependerá, principalmente, do teor de umidade, da classe do solo e da taxa de lotação animal (Marchão et al., 1996).

Dentre as técnicas mais utilizadas de quantificação da compactação do solo, citam-se: a pressão de pré-consolidação, também definida como capacidade de suporte de cargas, que é a máxima pressão que o solo suporta sem se deformar permanentemente (Dias Júnior, 1994) e o grau de compactação, que relaciona a densidade natural do solo com a máxima densidade que ele pode atingir (Dias Júnior \& Miranda, 2000; Oliveira et al., 2010). Pires et al., (2012) encontraram valores menores de pressão de pré-consolidação para áreas bem manejadas e com adubações adequadas.

O tráfego intenso de máquinas e pisoteio animal em solo com umidades que favorecem a compactação tem se tornado um fator preocupante para a manutenção de uma atividade agropecuária eficiente e conservacionista (Dias Júnior \& Pierce, 1996; Silva et al., 2002). Desse modo, torna-se fundamental entender a carga aplicada ao solo e a condição de umidade deste. Neste sentido objetivou-se com este estudo, gerar modelos de capacidade de suporte de carga para os diferentes usos e floresta nativa e através destes avaliar a degradação da estrutura do solo, definindo as pressões de pré-consolidação de cada área e a partir delas tomando medidas necessários para um melhor uso de cada área.

A hipótese estabelecida para o desenvolvimento desta pesquisa é de que os diferentes usos e floresta nativa podem alterar os atributos físicos e mecânicos do solo e degradar a estrutura do solo, afetando a pressão de pré-consolidação (PP) e o seu comportamento compressivo. 


\section{MATERIAL E MÉTODOS}

O experimento foi realizado na Fazenda experimental da Universidade Federal dos Vales do Jequitinhonha e Mucuri (UFVJM) situada em Curvelo, MG (Figura 1). O clima do município é considerado como tropical de altitude $\mathrm{Cwa}$, segundo a classificação de Köppen-Geiger, característica advinda de sua altitude média de 672 metros e da latitude de $18,45^{\circ} \mathrm{S}$ e longitude de $44,25^{\circ} \mathrm{W}$. Sendo assim, o período de verão registra chuvas e temperaturas elevadas, enquanto o inverno é seco com temperaturas mais baixas. O solo nesta área é classificado como Latossolo Vermelho Amarelo distrófico com textura média (Empresa Brasileira de Pesquisa Agropecuária, 2018).

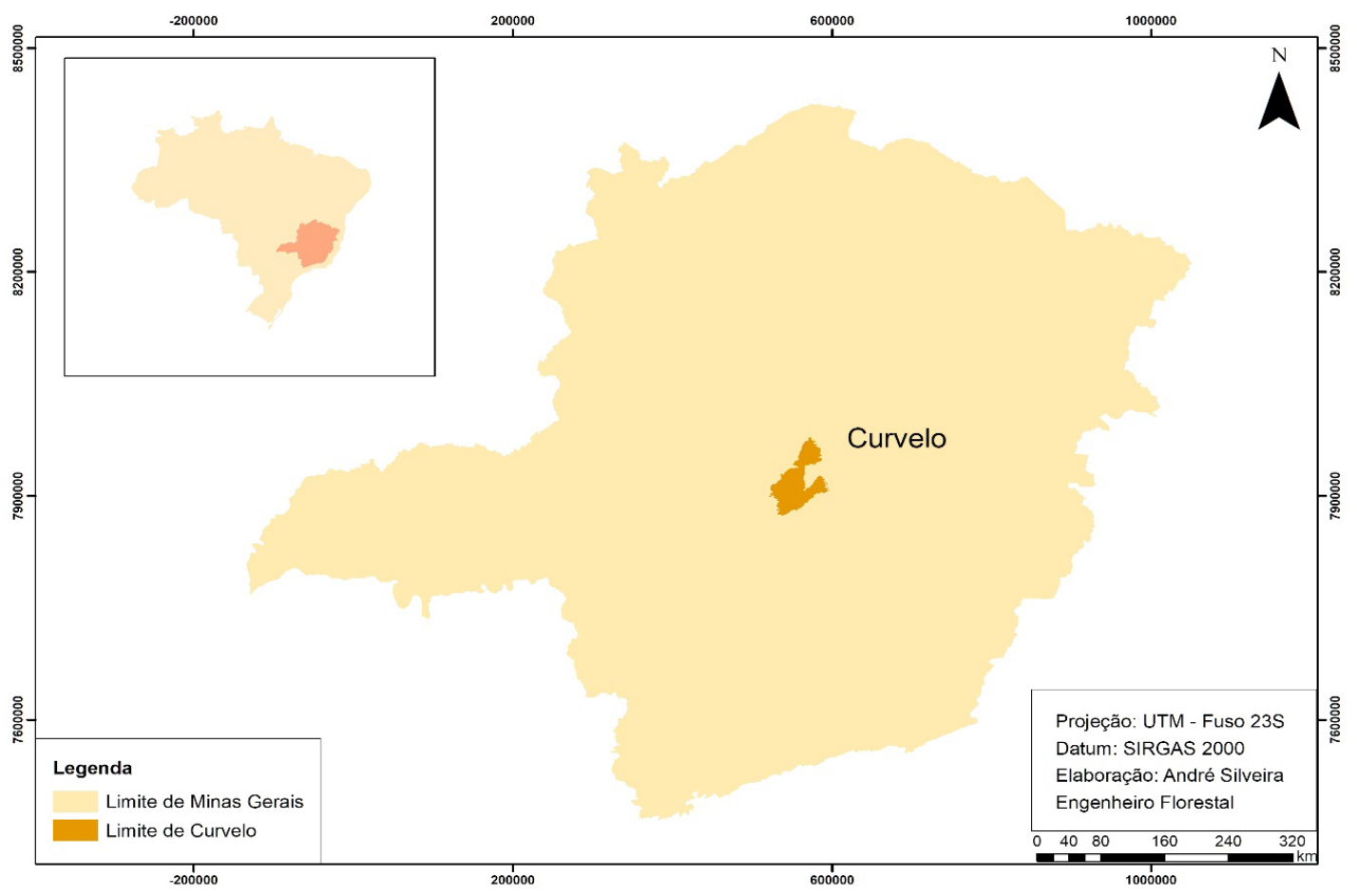

Figura 1. Localização da área de estudo.

O estudo foi conduzido nas seguintes áreas: CE do tipo campo limpo composto, FN e ILPF. O CE é composto principalmente pelas espécies: Burmanniaceae (Burmannia), Cyperaceae (Rhynchospora), Droseraceae (Drosera), Iridaceae (Cipura, Sisyrinchium), Lentibulariaceae (Utricularia), Lythraceae (Cuphea), Orchidaceae (Cleistes, Habenaria, Sarcoglottis), Poaceae (Aristida, Axonopus, Mesosetum, Panicum, Paspalum, Trachypogon), Caryocar brasiliense Cambess. e Polygalaceae (Polygala), com alguns sinais de degradação do solo, devido ao pisoteio animal sem controle no passado, causando processos erosivos. A FN, resquício de mata atlântica, é caracterizada por uma área preservada composta por uma sucessão avançada, onde as espécies principais são: Jaboticaba sabará (Plinia trunciflora), Mamica de Porca (Zanthoxylum rhoifolium), Cedro Rosa (Cedrela fissilis), Casca D'Anta (Rauvolfia sellowii), Fedegoso (Senna pendula), Jerivá (Syagrus romanzoffiana). A Integração Lavoura pecuária floresta (ILPF) implementada a 5 anos, é uma consorciação de Eucalipto urophylla $\times$ E. grandis com Brachiaria brizanta cv. MG5- Vitória. Complementando esse sistema, ocorre também o plantio de milho e a adubação é realizada segundo Ribeiro et al. (1999). Essa área, após a colheita do milho sofre pastejo de gado com taxa de lotação de 3,5 UA/ha. As amostras foram coletadas no período de outubro de 2019, sendo essas deformadas e indeformadas para a realização dos estudos de Pressão de pré-consolidação (PP), conforme descrito a seguir.

Com auxílio de um Amostrador de Uhland, foram coletadas 30 amostras indeformadas em cada área, com anéis volumétricos de $6,40 \mathrm{~cm}$ de diâmetro por $2,54 \mathrm{~cm}$ de altura. Essas amostras foram coletadas aleatoriamente a partir da camada superficial e subsuperficial. 
Na camada superficial de $0-5 \mathrm{~cm}$ foram coletadas 30 amostras, 10 amostras para cada área de estudo, seguindo a mesma metodologia para a camada subsuperficial de $20-40 \mathrm{~cm}$, totalizando 180 amostras indeformadas. As amostras foram envoltas em papel filme e parafinadas para não perderem umidade e nem serem danificadas no transporte. Em cada ponto de coleta foram também coletadas amostras deformadas de solos, para a análise de textura, matéria orgânica e umidade.

Todas as amostras foram levadas para o laboratório de física e mecânica dos solos da Universidade Federal dos Vales do Jequitinhonha e Mucuri. Foram realizadas também a análise granulométrica em amostras deformadas de solo, pelo método da pipeta (Day,1965; Empresa Brasileira de Pesquisa Agropecuária, 2017) e, do teor de matéria orgânica (Raji \& Quaggio, 1983). As amostras indeformadas foram condicionadas ao volume do solo contido equivalente ao volume dos anéis. Para a determinação da pressão de pré-consolidação (PP), as amostras foram saturadas por capilaridade em um período máximo de 72 horas e depois submetidas a um potencial matricial de $6 \mathrm{kPa}$ em um extrator de placas porosas de Richards. Uma vez estabilizadas, as amostras foram pesadas úmidas e encaminhadas para o ensaio de compressão uniaxial. A Tabela 1 apresenta a análise textural, valores de matéria orgânica e umidade na capacidade de campo dos locais avaliados.

Tabela 1. Análise textural, matéria orgânica e umidade na capacidade de campo para as situações estudadas.

\begin{tabular}{lccccc}
\hline Situações & Areia (\%) & Argila (\%) & Silte (\%) & $\begin{array}{c}\text { Matéria Orgânica } \\
\left(\mathbf{g ~ k g}^{-1}\right)\end{array}$ & $\begin{array}{c}\text { Umidade na } \\
\text { capacidade de } \\
\text { campo(kg kg-1) }\end{array}$ \\
\hline FN & 57,8 & 16,0 & 26,2 & 3,8 & 0,33 \\
CE & 57,9 & 16,0 & 26,1 & 1,8 & 0,30 \\
ILPF & 61,0 & 21,0 & 18,0 & 1,6 & 0,30 \\
& & & Camada 20-40 cm & & \\
FN & 57,3 & 18,3 & 24,4 & 3,2 & 0,34 \\
CE & 55,8 & 19,1 & 25,1 & 1,2 & 0,32 \\
ILPF & 57,3 & 22,9 & 19,8 & 1,4 & 0,32 \\
\hline
\end{tabular}

FN: Floresta Nativa; CE: Cerrado; ILPF: Integração Lavoura Pecuária e Floresta

Realizou-se ensaio de compressão uniaxial aplicando em cada amostra, 25, 50, 100, 200, 400,800 e $1600 \mathrm{kPa}$ de pressão, tendo sido a aplicação feita até que $90 \%$ da deformação máxima fosse alcançada (Dias Júnior \& Martins, 2017), submetendo-se logo a seguir uma nova pressão. Obteve-se as curvas de compressão do solo representando a pressão em escala logarítmica aplicada no eixo das abscissas, e a densidade do solo correspondente a cada pressão aplicada no eixo das ordenadas. Com base nas curvas de compressão do solo, foram obtidas as pressões de pré-consolidação $\left(\sigma_{p}\right)$ (Dias Júnior \& Martins, 2017). Os valores de pressão de pré-consolidação com os valores de umidade foram ajustados a uma regressão exponencial decrescente do tipo: $\sigma_{p}=10^{\left(a+b^{*} \times\right)}$ (Dias Júnior, 1994). As letras a e b representam parâmetros empíricos de ajuste do modelo, ou seja, o coeficiente linear e angular, respectivamente.

Foram realizadas as comparações entre os modelos de capacidade de suporte de carga utilizando o teste de homogeneidade de modelos lineares descrito em Snedecor \& Cochran (1989) e para a obtenção dos modelos de capacidade de suporte de carga do solo utilizou-se o software Sigma Plot 8.0 (2002). 


\section{RESULTADOS E DISCUSSÃO}

A Figura 2 apresenta as modelagens matemáticas e curvas geradas das duas alternativas de uso do solo e FN para as amostras coletadas. A partir dessas curvas nota-se que elas determinam a pressão de pré-consolidação em uma determinada umidade, e evidencia que são grandezas inversamente proporcionais, ou seja, quanto menor a umidade do solo maior será a sua pressão de pré-consolidação. Observa-se que solos mais secos apresentam maiores pressões de pré-consolidação e, consequentemente, maiores capacidades de suporte de cargas (Gontijo et al., 2011). Sendo assim, o uso do CE apresentou uma maior capacidade de suporte de carga do que o ILPF seguido pela FN.

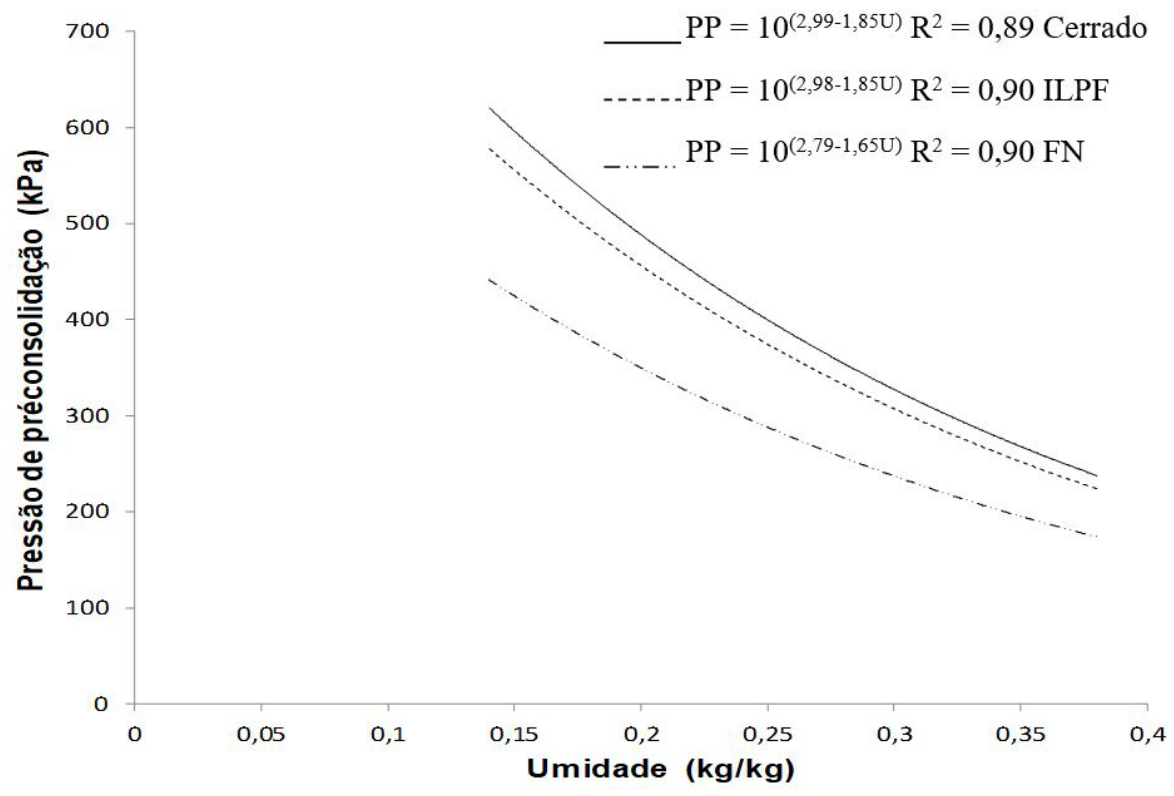

Figura 2. Modelagem da pressão de pré-consolidação em função da umidade em solos de CE, ILPF e FN.

Nota-se que os usos CE e ILPF apresentaram propriedades do solo bem semelhantes, ou seja, solos com o mesmo comportamento se encaixam em um único modelo para representálos. A Tabela 2 apresenta o resultado do teste de significância da comparação entre os modelos matemáticos gerados, onde o sistema ILPF e CE não diferem estatisticamente entre si pelo teste de Snedecor \& Cochran (1989) ao nível de significância de 5\%, sendo assim um único modelo representou os dois usos.

Tabela 2. Teste de significância de acordo com Snedecor \& Cochran (1989) entre as curvas compactação de um Latossolo Vermelho-Amarelo nas duas alternativas de uso do solo e FN.

\begin{tabular}{ccc}
\hline \multirow{2}{*}{ Usos e FN } & \multicolumn{2}{c}{$\mathbf{F}$} \\
\cline { 2 - 3 } & Coeficiente angular, b & Coeficiente linear, $\mathbf{a}$ \\
\hline FN vs ILPF & $\star \star$ & $\star \star$ \\
FN vS CE & $* \star$ & $\star *$ \\
ILPF vS CE & NS & NS
\end{tabular}

F: testa a homogeneidade dos dados; b coeficiente angular da regressão linearizada; a intercepto da regressão linearizada; NS: não significativo; ** significativo a 5\% de probabilidade respectivamente.

Uma vez não detectada a diferença entre a capacidade de suporte de cargas do CE e ILPF, os dados foram agrupados e uma nova modelagem foi ajustada (Figura 3). De posse desse novo modelo foram feitas as comparações entre eles, onde, CE e ILPF apresentaram uma maior capacidade de suporte de cargas que a FN. 


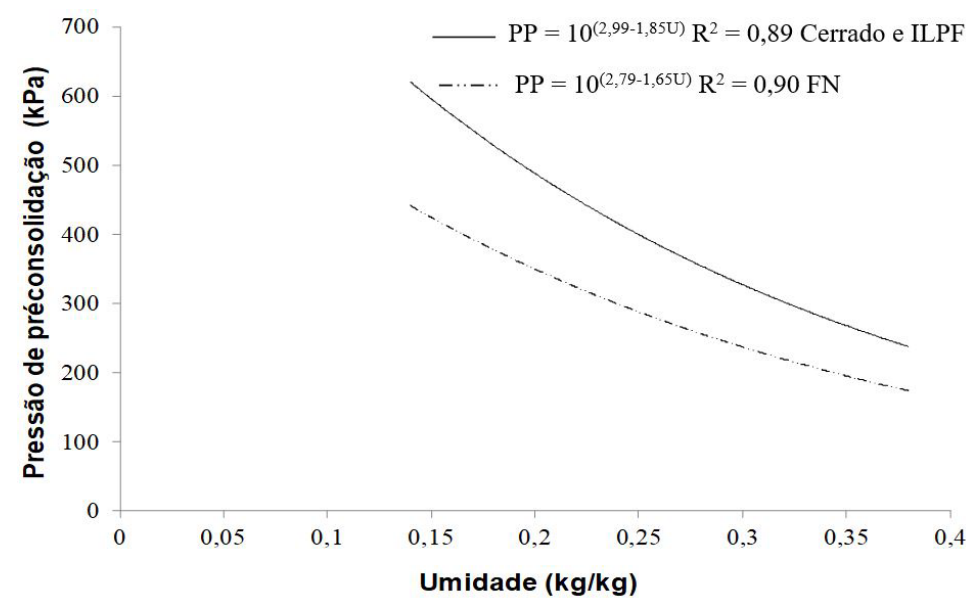

Figura 3. Modelagem da pressão de pré-consolidação em função da umidade.

A ausência de diferença estatística entre estas duas alternativas de uso do solo podem ser explicadas pela classe de solo ser a mesma, as áreas estarem muito próximas e ambas terem sofrido pisoteio animal por pelo menos um ano. Além disso, o sistema ILPF representado neste estudo, assim como $\circ \mathrm{CE}$, apresentaram baixa cobertura vegetal sobre 0 solo, fato que pode explicar também a ausência de diferença entre a capacidade de suporte de carga dessas duas situações, uma vez a cobertura vegetal agir como aliviador das cargas aplicadas sobre o solo (Pires et al., 2012).

A diferença estatística encontrada para o CE e o sistema ILPF em relação à $F N$, pode ser explicada pelo maior conteúdo de matéria orgânica (MO) deste último ambiente (Tabela 1), uma vez a MO promove uma diminuição na pressão de carga aplicada na estrutura do solo, deixando-o menos coeso, mais friável e com menor capacidade de suportar cargas (Rocha et al., 2007; Pires et al., 2012). Somado a isso, ressalta-se o fato de a FN não ter sofrido pisoteio animal e nem tráfego de máquinas, fatores estes preponderantes nesta menor capacidade de suporte de cargas.

Conforme a Figura 3, as áreas de ILPF e CE apresentaram maiores valores de pressão de préconsolidação (PP) em relação à FN. Esta maior resistência indica que provavelmente a estrutura do solo destes dois locais apresentaram deformação devido ao pisoteio (Pulido et al., 2017) e/ou tráfego de máquinas (Hamza \& Anderson, 2005). Estas áreas também responderam mais rápido à deformação, forçando a um ajuste acirrado das partículas do solo à carga aplicada.

A Tabela 3 apresenta os valores de pressão de pré-consolidação (PP) para as áreas de estudo, obtidas na umidade correspondente à capacidade de campo. Ao longo do ano nessas áreas de estudo, a faixa de umidade variou em torno de $0,2 \mathrm{~kg} \mathrm{~kg}^{-1}$. Assim, realizou-se uma simulação da capacidade de suporte de carga para as referidas, sendo seus valores apresentados na Tabela 3. A partir dessas pressões de pré-consolidação, pode-se definir limites de uso para cada área em cada época do ano, evitando assim danos físicos na estrutura do solo, prejuízos ambientais e econômicos em cada área de estudo.

Tabela 3. Pressão de pré-consolidação para as duas alternativas de uso do solo e FN na umidade da capacidade de campo e na umidade a $0,2 \mathrm{~kg} \mathrm{~kg}^{-1}$ do solo.

\begin{tabular}{cc}
\hline & Umidade na capacidade de campo -6kPa \\
\hline Usos do solo e FN & Pressão de pré-consolidação (kPa) \\
CE & $271 \mathrm{a}$ \\
ILPF & $268 \mathrm{a}$ \\
FN & $175 \mathrm{~b}$ \\
& Umidade em $0,2 \mathrm{~kg} \mathrm{~kg}^{-1}$ \\
Usos do solo e FN & Pressão de pré-consolidação (kPa) \\
CE & $415 \mathrm{a}$ \\
ILPF & 411 a \\
FN & $288 \mathrm{~b}$
\end{tabular}

Valores seguidos da mesma letra na vertical não diferem estatisticamente entre si pelo teste de Snedecor \& Cochran. 
Em valores reais, a pressão de pré-consolidação das áreas de ILPF e CE é muito superior a da FN, sendo o pisoteio animal considerado o fator mais importante nessa diferenciação. No Brasil, a média da pressão aplicada por tratores varia entre 200 e 300 kPa (Pires et al., 2012). Assim, o tráfego de máquinas nas referidas áreas não estaria comprometido, caso realizado na umidade próxima à capacidade de campo. Contudo, nessa mesma umidade do solo, o pisoteio animal fica restrito, pois a carga média aplicada por animais na área passa dos 400k Pa (Pires et al., 2012). Desse modo, sugere-se que o manejo animal nestes locais seja revisto, ou seja, ajustado à carga animal com a variável unidade animal mais adequada para a área e, também que, o pisoteio se dê com maior frequência na época mais seca. Na época mais úmida, por sua vez, a carga sobre o solo deve ser reduzida.

Ainda de acordo com a Tabela 3, em condições de baixa umidade, a capacidade de suporte de cargas aumenta, pois, o solo tem sua resistência ampliada pela aproximação das partículas (Larson et al., 1980). Nas áreas de CE e ILPF, em baixa umidade, o manejo animal não causaria danos à estrutura do solo, o qual resistiria à maioria das cargas aplicadas pelos animais. Porém, no caso de uma irrigação ou nos períodos chuvosos, a pressão de pastejo deve ser revista, considerando o número e/ou peso dos animais.

\section{CONCLUSÕES}

Os modelos de capacidade de suporte de carga se mostraram uma ferramenta muito eficaz na prevenção de danos físicos na estrutura solo. A pressão de pré-consolidação se mostrou uma excelente ferramenta, pois ela é a única propriedade preditiva das pressões a serem aplicadas ao solo para evitar que a compactação ocorra. Deve-se notar também que a pressão de pré-consolidação está totalmente relacionada com a umidade, onde, um solo mais seco suportou uma maior carga aplicada, sem ocorrer maiores danos. A coberta vegetal também exerceu um papel importante na capacidade de suporte de cargas, uma vez que, a matéria orgânica promove uma diminuição na capacidade de cargas do solo, deixando-o menos coeso e mais friável.

\section{REFERÊNCIAS}

Cambi, M., Hoshika, Y., Mariotti, B., Paoletti, E., Picchio, R., Venanzi, R., \& Marchi, E. (2017). Compaction by a forest machine affects soil quality and Quercus robur $\mathrm{L}$. seedling performance in an experimental field. Forest Ecology and Management, 384, 406-414. http://dx.doi.org/10.1016/j.foreco.2016.10.045.

Collares, G. L., Reinert, D. J., Reichert, J. M., \& Kaiser, D. R. (2011). Compactação superficial de Latossolos sob integração lavoura: pecuária de leite no noroeste do Rio Grande do Sul. Ciência Rural, 41(2), 246-250. http://dx.doi.org/10.1590/S0103-84782011000200011.

Day, P. R. (1965). Particle fractionation and particle-size analysis. In C. A. Black (Ed.), Methods of soil analysis: part 1 physical and mineralogical properties, including statistics of measurement and sampling (pp. 545-567).Madison: ASA/SSSA. .

Dias Júnior, M. S. (1994). Compression of three soils under long- term tillage and wheel traffic (114 p.). East Lansing: Michigan State University.

Dias Júnior, M. S., \& Martins, P. C. C. (2017). Ensaio de compressão uniaxial e modelos de capacidade de suporte de carga do solo. In Empresa Brasileira de Pesquisa Agropecuária (Org.), Manual de métodos de análise de solo (3. ed., pp. 153-172). Brasília: EMPRAPA.

Dias Júnior, M. S., \& Miranda, E. É. V. (2000). Comportamento da curva de compactação de cinco solos da região de Lavras (MG). Ciência e Agrotecnologia, 24, 337-346.

Dias Júnior, M. S., \& Pierce, F. J. (1996). O processo de compactação do solo e sua modelagem. Revista Brasileira de Ciência do Solo, 20, 175-182.

Empresa Brasileira de Pesquisa Agropecuária. (2017). Manual de métodos de análise de solo (3. ed., 577 p.). Brasília: EMBRAPA.

Empresa Brasileira de Pesquisa Agropecuária. (2018). Centro Nacional de Pesquisas do Solo. Sistema brasileiro de classificação de solos (5. ed., 355 p.). Brasília: Embrapa Solos. 
Epron, D., Plain, C., Ndiaye, F., Bonnaud, P., Pasquier, C., \& Ranger, J. (2016). Effects of compaction by heavy machine traffic on soil fluxes of methane and carbon dioxide in a temperate broadleaved forest. Forest Ecology and Management, 382, 1-9. http://dx.doi.org/10.1016/j.foreco.2016.09.037.

Gontijo, I., Dias Junior, M. S., Guimarães, P. T. G., Oliveira, M. S., \& Ajayi, A. E. (2011). Spatial patterns of preconsolidation pressure and soil moisture along transects in two directions under coffee. Revista Brasileira de Ciência do Solo, 35(4), 1189-1196. http://dx.doi.org/10.1590/S0100-06832011000400012.

Gubiani, P. I., Reichert, J. M., \& Reinert, D. J. (2013). Indicadores hídrico-mecânicos de compactação do solo e crescimento de plantas. Revista Brasileira de Ciência do Solo, 37(1), 1-10. http://dx.doi.org/10.1590/S0100-06832013000100001.

Hamza, M. A., \& Anderson, W. K. (2005). Soil compaction in cropping systems: a review of the nature, causes and possible solutions. Soil \& Tillage Research, 82(2), 121-145. http://dx.doi.org/10.1016/j.still.2004.08.009.

Kunz, M., Gonçalves, A. D. M. A., Reichert, J. M., Guimarães, R. M. L., Reinert, D. J., \& Rodrigues, M. F. (2013). Compactação do solo na integração soja-pecuária de leite em Latossolo argiloso com semeadura direta e escarificação. Revista Brasileira de Ciência do Solo, 37(6), 1699-1708. http://dx.doi.org/10.1590/S0100-06832013000600026.

Larson, W. E., Gupta, S. C., \& Useche, R. A. (1980). Compression of agricultural soils from eight soils orders. Soil Science Society of America Journal, 44(3), 450-457. http://dx.doi.org/10.2136/sssaj1980.03615995004400030002x.

Lima, J. S. S., \& Leite, A. M. P. (2014). Mecanização. In C. C. Machado (Ed.), Colheita Florestal (3. ed., Cap. 2, pp. 42-72). Viçosa: UFV.

Lopes, E. S., Sampietro, J. A., Pereira, A. L. N., \& Oliveira, D. (2011). Compactação de um Latossolo submetido ao tráfego de skidder com diferentes rodados. Floresta, 41(3), 471-480. http://dx.doi.org/10.5380/rf.v41i3.24038.

Lopes, S. E., Fernandes, H. C., Vieira, L. B., Machado, C. C., \& Rinaldi, P. C. N. (2006). Compactação de um solo de uso florestal submetido ao tráfego de arraste de madeira. Revista Árvore, 30(3), 369-376. http://dx.doi.org/10.1590/S0100-67622006000300007.

Marchão, R. L., Balbino, L. C., Silva, E. M., \& Santos Júnior, J. D. G. (1996). Qualidade física de um latossolo vermelho sob sistemas de integração lavoura-pecuária no cerrado. Pesquisa Agropecuária Brasileira, 31(3), 221-232.

Oliveira, V. S., Rolim, M. M., Vasconcelos, R. F. B., Costa, Y. D. J., \& Pedrosa, E. M. R. (2010). Compactação de um argissolo amarelo distrocoeso submetido a diferentes manejos. Revista Brasileira de Engenharia Agrícola e Ambiental, 14(9), 914-920. http://dx.doi.org/10.1590/S1415-43662010000900002.

Pires, B. S., Dias Júnior, M. S., Rocha, W. W., Araujo Junior, C. F., \& Carvalho, R. C. R. (2012). Modelos de capacidade de suporte de carga de um Latossolo Vermelho-Amarelo sob diferentes usos e manejos. Revista Brasileira de Ciência do Solo, 36(2), 635-642. http://dx.doi.org/10.1590/S0100-06832012000200032.

Pulido, M., Schnabel, S., Contador, J. F. L., Lozano-Parra, J., Gómez-Gutiérrez, Á., Brevik, E. C., \& Cerdà, A. (2017). Reduction of the frequency of herbaceous roots as an effect of soil compaction induced by heavy grazing in rangelands of SW Spain. Catena, 158, 381-389. http://dx.doi.org/10.1016/j.catena.2017.07.019.

Raji, B., \& Quaggio, J. A. (1983). Métodos de análise de solo para fins de fertilidade (21 p.). Campinas: Instituto Agronômico de Campinas.

Ribeiro, A. C., Guimarães, P. T., \& Alvarez V. V. H. (1999). Recomendações para o uso de corretivos e fertilizantes em minas gerais (358 p.). Viçosa: Comissão de fertilidade do solo do estado de Minas gerais-CFSEMG.

Rocha, W. W., Borges, S. R., Victória, E. P., \& Nunes, A. B. (2007). Resistência ao cisalhamento do solo do ponto de vista ambiental. In M. H. N. Abreu (Ed.), Ciências Ambientais: abordagem multidisciplinar (pp. 87-124.). Belo Horizonte: UEMG.

Secco, D., Reinert, D. J., Reichert, J. M., \& Silva, V. R. (2009). Atributos físicos e rendimento de grãos de trigo, soja e milho em dois Latossolos compactados e escarificados. Ciência Rural, 39(1), 58-64. http://dx.doi.org/10.1590/S0103-84782009000100010.

Silva, V. R., Reinert, D. J., \& Reichert, J. M. (2002). Fatores controladores da compressibilidade de um Argissolo Vermelho-Amarelo distrófico arênico e de um Latossolo Vermelho distrófico típico. II Grau de saturação em água. Revista Brasileira de Ciência do Solo, 26(1), 9-15. http://dx.doi.org/10.1590/S0100-06832002000100002.

Smucker, A. J. M., \& Erickson, A. E. (1989). Tillage and compactive modifications of gaseous flow and soil aeration. In W. E. Larson, G. R. Blake, R. R. Allmaras, W. B. Voorhees \& S. C. Gupta (Eds.), Mechanics related process in structured agricultural soils (NATO Applied Sciences, Vol. 172, pp. 205-221). Dordrecht: Springer. . http://dx.doi.org/10.1007/978-94-009-2421-5_16. 
Snedecor, G. W., \& Cochran, W. G. (1989). Statistical methods (8th ed.). Ames: lowa State University.

Tang, A. M., Cui, Y., Richard, G., \& Défossez, D. (2011). A study on the air permeability as affected by compression of three French soils. Geoderma, 162(1-2), 171-181. http://dx.doi.org/10.1016/j.geoderma.2011.01.019.

Contribuição dos Autores: ARS: Conceituação, Curadoria de Dados, Análise Formal, Metodologia, Supervisão, Validação, Visualização, Escrita - Primeira Redação, Escrita - Revisão e Edição; WWR: Conceituação, Curadoria de Dados, Metodologia, Escrita - Revisão e Edição; AMPL: Revisão e Edição; JJS: Revisão e Edição; MSDJ: Revisão e Edição. 